\title{
CENTRIOLES IN SYMMETRIC SPACES
}

\author{
PETER QUAST
}

\section{In honor of Ernst A. Ruh on the occasion of his 75th birthday}

\begin{abstract}
We describe all centrioles in irreducible simply connected pointed symmetric spaces of compact type in terms of the root system of the ambient space, and we study some geometric properties of centrioles.
\end{abstract}

\section{$\S 0$. Introduction}

Symmetric spaces are generalizations of spaces of constant sectional curvature. Though symmetric spaces need not have constant sectional curvature, their sectional curvatures remain constant under parallel translations of 2-dimensional tangent planes along regular curves. Thus, the easiest examples of simply connected compact symmetric spaces are spheres. The most prominent totally geodesic submanifolds in spheres are equatorial hyperspheres. Several generalizations of these submanifolds to other ambient symmetric spaces have been suggested, for example, embedded minimal hyperspheres (see $[\mathrm{HH}],[\mathrm{HHT}]$ ).

In this paper, we study another generalization of equatorial hyperspheres in spheres called centrioles. Centrioles, a term borrowed from cytology, have been introduced by Chen and Nagano [CN2]. Centrioles in symmetric spaces arise in the same way as equatorial hyperspheres in spheres, namely, as connected components of the midpoint locus of all geodesic arcs joining two antipodal points. They share some nice properties with equatorial hyperspheres; for example, they are orbits of isotropy groups, and they are reflective in the sense of Leung [Le]: they are connected components of the fixedpoint set of involutive isometries of the ambient space (see Section 1).

Centrioles also play an important role in Bott's original proof (see [Bt]) of his periodicity theorem for the homotopy of classical Lie groups (see

Received May 28, 2011. Revised May 19, 2012. Accepted May 24, 2012.

First published online April 26, 2013.

2010 Mathematics Subject Classification. Primary 53C40; Secondary 53C35. 
also [Ml]). They can also be used to calculate homotopy groups of some exceptional symmetric spaces, for example, of $\mathrm{E}_{7} /\left(S^{1} \mathrm{E}_{6}\right)$ (see [Bu3], [Bu1], [Mi1], [Mi2], and [Q1]), and to study the periodicity of certain standard inclusions (see [MQ2]).

In contrast to spheres, compact symmetric spaces of higher rank with a chosen base point may admit several antipodal points and different nonisomorphic centrioles. Moreover, not all centrioles consist necessarily of midpoints of shortest geodesic arcs between these antipodal points.

Our article is organized in the following manner.

Section 1 is devoted to the beautiful and rich geometry of centrioles.

In Section 2, we provide a complete description of centrioles in irreducible simply connected symmetric spaces of compact type in terms of the root system of the ambient space (see Theorems 12 and 20).

Hyperspheres in spheres are maximal proper totally geodesic submanifolds, and in Section 3 we show that this still holds for s-centrioles (see Theorem 30).

To make this article more self-contained, we end with an appendix, in which we collect some well-known facts about root systems needed in this paper.

\section{$\S 1$. The geometry of centrioles}

\subsection{Definitions}

Let $P$ be a compact Riemannian symmetric space. (We always assume that $P$ is connected.) If we choose a base point $o$, we call the pair $(P, o)$ a pointed symmetric space. Next, we generalize the notion of antipode in a pointed round sphere. Following Chen and Nagano [CN2], we call a point $p \in P$ different from $o$ a pole of $(P, o)$ if $s_{o}=s_{p}$. Here, $s_{o}$ and $s_{p}$ denote the geodesic symmetries of $P$ at the points $o$ and $p$, respectively.

Let $p$ be a pole of $(P, o)$. The centrosome $C(o, p)$ of $(P, o)$ relative to $p$ is the set of the midpoints of all geodesic arcs in $P$ between $o$ and $p$. A connected component of a centrosome is called a centriole (see [CN2]). If $x$ is a point in $C(o, p)$, we denote by $C_{x}(o, p)$ the centriole containing $x$.

For further use, we decide on the following nomenclature. A geodesic $\gamma: \mathbb{R} \rightarrow P$ joins the point $x \in P$ to the point $y \in P$ if $\gamma(0)=x$ and $\gamma(1)=y$.

The transvection group $\mathrm{G}$ of a symmetric space $P$ is the closed subgroup of the isometry group $\mathrm{I}(P)$ of $P$ (in the compact-open topology) generated by the products of two geodesic symmetries of $P$. If $P$ is of compact type, 
that is, if the universal Riemannian cover of $P$ is still compact, then $\mathrm{G}$ is the identity component of $\mathrm{I}(P)$ (see, e.g., [W], [He]). Since $P$ is connected, the transvection group of $P$ acts transitively on $P$. Indeed, let $x$ and $y$ be two points in $P$, and let $\gamma$ be a geodesic in $P$ joining $x$ to $y$. To this geodesic corresponds the 1-parameter subgroup

$$
\tau_{\gamma}: \mathbb{R} \rightarrow \mathrm{G}, \quad t \mapsto s_{\gamma(t / 2)} \circ s_{\gamma(0)}
$$

of transvections along $\gamma$ (see, e.g., [S3, p. 175]). We have $y=\tau_{\gamma}(1) . x$, where we denote by $g . x$ the image of $x$ under the isometry $g$.

\subsection{Geometric properties}

In this section, we study some nice geometric properties of centrioles. Though most of the presented results are known or folklore (see, e.g., [CN2], [N1], [N2], [Ch1]), we discuss them and provide geometric proofs for the sake of completeness.

Lemma 1 ([CN2, Proposition 2.9], [Ch2, Theorem 3.3]). For any pole $p$ of a compact pointed symmetric space $(P, o)$, there exists a unique fixedpoint-free involutive isometry $\rho_{p}$ of $P$ that maps o to $p$ and commutes with all transvections of $P$. Moreover, the orbit space $P / \Gamma_{p}$ with $\Gamma_{p}:=\left\{\mathrm{Id}, \rho_{p}\right\}$ is a symmetric space.

Proof. (The outline of this proof can be found in [CN2, proof of Proposition 2.9] or [Ch2, proof of Theorem 3.3]). It is well known that the pointed Cartan map

$$
\iota: P \rightarrow \mathrm{G}, \quad p \mapsto s_{p} \circ s_{o}
$$

is a covering map onto its image, and this image is again a symmetric space. According to [W, p. 244], there exists a discrete subgroup $\Gamma$ of the centralizer $\Delta=C_{\mathrm{G}}(\mathrm{I}(P))$ of $\mathrm{G}$ in the isometry group $\mathrm{I}(P)$ of $P$ such that the image of $\iota$ is isomorphic to $P / \Gamma$ as a symmetric space (for a suitable bi-invariant metric on $\mathrm{G}$ ).

Since $\Gamma$ is the deck transformation group of the covering map $\iota$ (see, e.g., [Ha, Proposition 1.40]), every nontrivial element of $\Gamma$ acts fixed-point-free. Since $\iota(o)=\iota(p)$ holds by the definition of a pole, there must be a unique element $\rho_{p}$ in $\Gamma$ satisfying $\rho_{p}(o)=p$.

Any geodesic $\gamma$ in $P$ that joins $o$ to $p$ satisfies $\gamma(2)=s_{p}(o)=s_{o}(o)=o$. Let $\tau_{\gamma}$ be the 1-parameter subgroup of transvections along $\gamma$; then $\tau_{\gamma}(1)$ maps 
$\gamma(0)=o$ onto $\gamma(1)=p$ and squares to the identity, because $\tau_{\gamma}(1) \circ \tau_{\gamma}(1)=$ $\tau_{\gamma}(2)=s_{\gamma(1)} \circ s_{\gamma(0)}=s_{p} \circ s_{o}=s_{o}^{2}=\mathrm{Id}$.

Since $\rho_{p}$ commutes with any transvection, we get $\rho_{p}^{2} . o=\rho_{p} \cdot p=$ $\rho_{p} \cdot\left(\tau_{\gamma}(1) . o\right)=\tau_{\gamma}(1) \cdot\left(\rho_{p} . o\right)=\tau_{\gamma}(1) \cdot\left(\tau_{\gamma}(1) . o\right)=\tau_{\gamma}(2) . o=o$. Hence, $\rho_{p}^{2}$ is an element of $\Gamma$ that has a fixed point. Thus, $\rho_{p}^{2}=$ Id. This also shows that $\Gamma_{p}:=\left\{\mathrm{Id}, \rho_{p}\right\}$ is a subgroup of $\Delta$ which is isomorphic to $\mathbb{Z}_{2}$. The result in [W, p. 244] implies that $P / \Gamma_{p}$ is a symmetric space.

The next proposition shows that centrioles are not just totally geodesic submanifolds, they are reflective submanifolds in the sense of Leung [Le] (see [N1, Definition and Proposition 2.12] for the statement); that is, they are connected components of the fixed-point set of an involutive isometry of the ambient symmetric space $P$.

Proposition 2 ([N1, Definition and Proposition 2.12(ii)]). Centrioles of connected compact pointed symmetric spaces are reflective submanifolds. More precisely, the centrosome $C(o, p)$ is the fixed-point set of the involutive isometry $r_{p}:=\rho_{p} \circ s_{o}$.

In particular, centrioles are totally geodesic submanifolds.

Proof. Let $p$ be a pole of $(P, o)$, and let $x \in C(o, p)$ be the midpoint of a geodesic arc $\gamma$ in $P$ joining $\gamma(0)=o$ to $\gamma(1)=p$. Then $\tilde{\gamma}:=\rho_{p} \circ \gamma$ is again a geodesic in $P$ and satisfies $\tilde{\gamma}(0)=p$ and $\tilde{\gamma}(1)=o$. Let $\pi_{p}: P \rightarrow P / \Gamma_{p}$ be the canonical projection; then $\pi_{p} \circ \gamma=\pi_{p} \circ \tilde{\gamma}$. Hence, $\tilde{\gamma}(t)=\gamma(t+1)$. Thus, $r_{p} \cdot x=r_{p} \cdot \gamma(1 / 2)=\rho_{p} \cdot\left(s_{o} \cdot \gamma(1 / 2)\right)=\rho_{p} \cdot \gamma(-1 / 2)=\tilde{\gamma}(-1 / 2)=\gamma(1 / 2)=x$.

Conversely, let $x$ be a fixed point of $r_{p}$. Since $\rho_{p}$ is involutive, we get $\rho_{p} . x=$ $\rho_{p} \cdot\left(r_{p} . x\right)=\left(\rho_{p} \circ \rho_{p}\right) \cdot\left(s_{o} . x\right)=s_{o} . x$. Let $\gamma$ be a geodesic in $P$ satisfying $\gamma(0)=$ $o$ and $\gamma(1 / 2)=x$. Then $\pi_{p}(\gamma(1 / 2))=\pi_{p}\left(\rho_{p} . x\right)=\pi_{p}\left(s_{o} \cdot x\right)=\pi_{p}(\gamma(-1 / 2))$. Since geodesics in symmetric spaces are orbits of 1-parameter groups of isometries, they close at any self-intersection. Thus, $\left(\pi_{p} \circ \gamma\right)(t)=\left(\pi_{p} \circ \gamma\right)(t+$ $1)$, and in particular, $\left(\pi_{p} \circ \gamma\right)(0)=\left(\pi_{p} \circ \gamma\right)(1)$. Hence, either $\gamma(1)=\gamma(0)=o$ or $\gamma(1)=p$. The first equation implies that $\gamma(t)=\gamma(t+1)$, and hence $x=$ $\gamma(1 / 2)=\gamma(-1 / 2)=s_{o} \cdot x=\rho_{p} . x$. This contradicts the fact that $\rho_{p}$ has no fixed point. Thus, $\gamma(1)=p$, and $x$ lies in $C(o, p)$.

To prove that $r_{p}$ is an involution, we actually show that $s_{o} \circ \rho_{p} \circ s_{o}=\rho_{p}$. Since $\rho_{p}$ commutes with any transvection, we get $\left(s_{o} \circ \rho_{p} \circ s_{o}\right) \circ\left(s_{p} \circ s_{q}\right)=$ $s_{o} \circ \rho_{p} \circ\left(s_{o} \circ s_{p}\right) \circ s_{q}=s_{o} \circ\left(s_{o} \circ s_{p}\right) \circ \rho_{p} \circ s_{q}=s_{p} \circ \rho_{p} \circ s_{q} \circ s_{o} \circ s_{o}=\left(s_{p} \circ\right.$ $\left.s_{q}\right) \circ\left(s_{o} \circ \rho_{p} \circ s_{o}\right)$ for all points $p$ and $q$ in $P$. Since the transvection group $\mathrm{G}$ of $P$ is generated by all products of two geodesic symmetries of $P$, we see 
that $s_{o} \circ \rho_{p} \circ s_{o}$ centralizes G. Since $\rho_{p}$ is an involution without fixed points, the same holds true for $s_{o} \circ \rho_{p} \circ s_{o}$. Moreover, $\left(s_{o} \circ \rho_{p} \circ s_{o}\right) . o=p$. Lemma 1 yields $s_{o} \circ \rho_{p} \circ s_{o}=\rho_{p}$ by uniqueness.

Observation 3. Let $C(o, p)$ be the centrosome of $(P, o)$ corresponding to $p$, and let $r_{p}$ be the corresponding reflection defined in Proposition 2. Then $r_{p}(o)=p$.

Each point $x \in P$ induces an involutive automorphism

$$
\sigma_{x}: \mathrm{G} \rightarrow \mathrm{G}, \quad g \mapsto s_{x} \circ g \circ s_{x}
$$

of the transvection group G. Its differential $\left(\sigma_{x}\right)_{*}$ at the identity induces an involutive automorphism of the Lie algebra $\mathfrak{g}$ of $\mathrm{G}$. It is well known (see, e.g., [He, Chapter IV, Section 3]) that the fixed-point set of $\left(\sigma_{x}\right)_{*}$ is the Lie algebra of the stabilizer of $x$ in G.

If $p$ is a pole of $(P, o)$, then $\sigma_{o}=\sigma_{p}$. Hence, the identity components of the stabilizers of $o$ and of $p$ in $\mathrm{G}$ coincide, since they have the same Lie algebras. We denote this connected subgroup of $\mathrm{G}$ by $\mathrm{K}_{e}$. The following result is known (see, e.g., [Ch1, Proposition 5.1] and [MQ2, Lemma 2.7]).

LEMma 4. Every centriole of $C(o, p)$ is a $\mathrm{K}_{e}$-orbit.

Proof. The method of the proof presented here can also be found in [MQ2, Lemma 2.7]. It is similar in spirit to [Ch1, proof of Lemma 3.4].

Let $x$ be a point in $C(o, p)$, and let $C_{x}(o, p)$ be the centriole containing it. We have to show that $\mathrm{K}_{e} \cdot x=C_{x}(o, p)$.

To show that $\mathrm{K}_{e} \cdot x \subset C_{x}(o, p)$, we take a geodesic arc $\gamma:[0,1] \rightarrow P$ that satisfies $\gamma(0)=o, \gamma(1 / 2)=x$, and $\gamma(1)=p$ and an element $k \in \mathrm{K}_{e}$. Since $k$ stabilizes both $o$ and $p$, the geodesic arc $\tilde{\gamma}:=k \circ \gamma$ satisfies $\tilde{\gamma}(0)=o$ and $\tilde{\gamma}(1)=p$. Hence, $\tilde{\gamma}(1 / 2)$ lies in $C(o, p)$. This shows that $\mathrm{K}_{e} \cdot x \subset C(o, p)$. Since $\mathrm{K}_{e} \cdot x$ is connected, we conclude that $\mathrm{K}_{e} \cdot x \subset C_{x}(o, p)$.

To show the opposite inclusion (namely, that $C_{x}(o, p) \subset \mathrm{K}_{e} . x$ ), we take a point $y \in C_{x}(o, p)$. Since $C_{x}(o, p)$ is connected and closed and therefore compact, there is a geodesic $c: \mathbb{R} \rightarrow C_{x}(o, p)$ in $C_{x}(o, p)$ satisfying $c(0)=x$ and $c(1)=y$.

Since $C_{x}(o, p)$ is a totally geodesic submanifold of $P, c$ is also a geodesic in $P$. Let $\tau_{c}$ be the 1-parameter subgroup of transvections in $P$ along $c$. For any $t_{0} \in \mathbb{R}$, the isometry $\tau_{c}\left(t_{0}\right)$ stabilizes both $o$ and $p$. Indeed, since $x$ and 
$c\left(t_{0} / 2\right)$ are midpoints of geodesic arcs in $P$ joining $o$ to $p$, we have $s_{x} . o=$ $p, s_{c\left(t_{0} / 2\right)} \cdot p=o$ and $s_{x} \cdot p=o, s_{c\left(t_{0} / 2\right)} \cdot o=p$, respectively. Thus, $\tau_{c}\left(t_{0}\right) . o=o$ and $\tau_{c}\left(t_{0}\right) \cdot p=p$. Since $\tau_{c}(0)$ is the identity, we see that $\tau_{c}$ takes values in $K_{e}$.

Now $y=\tau_{c}(1) . x \in \mathrm{K}_{e} . x$. This shows the other inclusion.

Corollary 5. Let $x \in C(o, p)$, and let $\gamma: \mathbb{R} \rightarrow P$ be a geodesic in $P$ satisfying $\gamma(0)=o$ and $\gamma(1 / 2)=x$. Then $\gamma$ intersects $C(o, p)$ perpendicularly in $x$; that is, $\dot{\gamma}(1 / 2) \perp T_{x} C(o, p)$.

Proof. By Lemma 4, the centriole $C_{x}(o, p)$ equals $\mathrm{K}_{e} \cdot x=\mathrm{K}_{e} \cdot \gamma(1 / 2)$. Since all geodesic arcs in $\left\{\left.k \cdot \gamma\right|_{[0,(1 / 2)]}: k \in \mathrm{K}_{e}\right\}$ have the same length and energy, our claim follows from the first variation formula for the length or the energy (see, e.g., [S3, p. 89]).

Corollary 6. Let $x \in C(o, p)$, and let $\gamma: \mathbb{R} \rightarrow P$ be a geodesic in $P$ satisfying $\gamma(0)=o$ and $\gamma(1 / 2)=x$. Then $\gamma(1)=p$.

Proof. Since $r_{p}$ is an involutive isometry whose fixed-point set is $C(o, p)$, its differential $D_{x} r_{p}$ at $x$ is an involutive linear isometry of $T_{x} P$ whose fixedpoint set is $T_{x} C(o, p)$ and whose $(-1)$-eigenspace is $\left(T_{x} C(o, p)\right)^{\perp}$. Corollary 5 implies that $D_{x} r_{p}(\dot{\gamma}(1 / 2))=-\dot{\gamma}(1 / 2)$. Thus, $\left(r_{p} \circ \gamma\right)(t)=\gamma(1-t)$, and therefore, $\gamma(1)=\gamma(1-0)=\left(r_{p} \circ \gamma\right)(0)=r_{p} . o=p$ by Observation 3 .

\section{$\S 2$. A classification of centrioles}

In this section, we describe all centrioles of an irreducible pointed simply connected symmetric space of compact type. We refer the reader to our appendix for details on the notation and terminology used in this section and for a brief overview on root systems. Further details can be found in the classical literature (e.g., [He], [Lo]).

\subsection{The center of a symmetric space of compact type}

Let us first assume that $P$ is an irreducible symmetric space of compact type (not yet necessarily simply connected). To admit centrioles, the pointed symmetric space $(P, o)$ must of course have poles. Lemma 1 shows that in this case $P$ must cover another symmetric space. This means in particular that $P$ cannot be an adjoint space. The adjoint space $\operatorname{Ad}(P)$ of $P$ is the (up to isometry) unique symmetric space that is locally isometric to $P$ and that has the following property: $\operatorname{Ad}(P)$ does not cover properly any other symmetric space (see [He, p. 327]). We can describe the adjoint space as an orbit space. As in the proof of Lemma 1 , we denote by $\Delta:=C_{\mathrm{G}}(\mathrm{I}(P))$ the 
centralizer of $\mathrm{G}$ in the isometry group of $P$. Then $\operatorname{Ad}(P)$ can be identified with the orbit space $P / \Delta$ (see [W, p. 244]).

To admit poles and centrioles, $P$ cannot be $\operatorname{Ad}(P)$. In particular, $\operatorname{Ad}(P)$ is not simply connected in this case. The description of the fundamental group of $\operatorname{Ad}(P)$ due to Cartan $[\mathrm{C}]$ and Takeuchi $[\mathrm{Tk}]$ shows that the tangent Lie triple $\mathfrak{p}$ of $(P, o)$ must contain nonzero elements $X$ with the property that $\operatorname{ad}(X)^{3}=-\operatorname{ad}(X)$ (see also [MQ1]). We call these elements extrinsically symmetric, because their isotropy orbits are extrinsically symmetric submanifolds in the Euclidean space $\mathfrak{p}$ (see [F2], [EH]). Extrinsically symmetric elements in $\mathfrak{p}$ exist if and only if a simple root system representing a fundamental Weyl chamber in $\mathfrak{p}$ contains a root whose coefficient in the expansion of the highest root is 1 (see, e.g., [MQ1, Lemma 2.1] and [KN]). Looking at the list of possible root systems (see, e.g., [He, Chapter X]), we can make the following observation.

OBSERVATION 7. To admit centrioles, the root system of $P$ must allow for extrinsically symmetric elements. Therefore, it can only be of type $\mathfrak{a}_{r}$, $\mathfrak{b}_{r}, \mathfrak{c}_{r}, \mathfrak{d}_{r}(r \geq 4), \mathfrak{e}_{6}$, or $\mathfrak{e}_{7}$. In particular, the root system of $P$ is reduced.

To determine all centrioles, we need a description of the poles of a pointed symmetric space $(P, o)$. Poles are special points in the center $Z(P, o)$ of $(P, o)$. If $\pi: P \rightarrow P / \Delta \cong \operatorname{Ad}(P)$ denotes the canonical projection, then the center $Z(P, o)$ of $(P, o)$ is the preimage of $\pi(o)$; that is,

$$
Z(P, o):=\pi^{-1}(\pi(o)) .
$$

Proposition 8 ([Lo, p. 64, Proposition 2.1.b]). We have

$$
Z(P, o)=\left\{x \in P: \mathrm{K}_{e} \cdot x=x\right\} .
$$

\subsection{Poles and polars}

By Lemma 1 , any pole $p$ of $(P, o)$ lies in the center of $(P, o)$. To characterize poles of $(P, o)$ among the center elements, we first notice that poles are special examples of polars, namely, singleton polars. A polar in $(P, o)$ is a connected component of the set of all midpoints of simple closed geodesics in $P$ that emanate at the base point $o$-in other words, a connected component of the fixed-point set of the geodesic symmetry $s_{o}$. Polars have been introduced and classified by Chen and Nagano [CN1]. (See also [Ch1] for a survey and [N1] for more details about the classification of polars.) Along the lines of the proof of Lemma 4, one can show that polars of $(P, o)$ are $\mathrm{K}_{e}$-orbits (see [CN1] or [Ch1, Lemma 3.4]). We conclude with the following. 
Observation 9. The set of poles of $(P, o)$ is the intersection of the center of $(P, o)$ with the union of all polars of $(P, o)$.

Proof. If $p$ is a pole of $(P, o)$, then $p$ lies in $Z(P, o) \backslash\{o\}$ by Lemma 1 . Moreover, let $\gamma$ be a shortest geodesic in $P$ joining $o=\gamma(0)$ to $p=\gamma(1)$; then $o=s_{p} . o=\gamma(2)$. Hence, $p$ is the midpoint of a simple closed geodesic emanating at $o$.

Conversely, let $p \in Z(P, o)$ be the midpoint of some simple closed geodesic emanating at $o$. Then $p$ lies in the polar $\mathrm{K}_{e} \cdot p$ of $(P, o)$. By Proposition 8, this polar is a singleton which is different from $\{o\}$. Thus, $p$ is a pole.

\subsection{Lattices}

In this section, we look at the initial directions of geodesics in $P$ joining $o$ to a pole or to a point in $Z(P, o)$. Since these points are fixed under the action of $\mathrm{K}_{e}$, we may assume that the initial direction lies in some fixed maximal abelian subspace $\mathfrak{a}$ of $\mathfrak{p}$.

The center lattice in $\mathfrak{a}$ is defined by

$$
\Gamma_{Z}(P, o):=\{X \in \mathfrak{a}: \exp (X) . o \in Z(P, o)\},
$$

where $\exp : \mathfrak{g} \rightarrow \mathrm{G}$ denotes the Lie-theoretic exponential map.

To describe this lattice in terms of roots, let $\Omega_{P}$ be the root system of $P$ corresponding to $\mathfrak{a}$. By Observation 7 , we may assume that $\Omega_{P}$ is reduced. It is well known (see, e.g., [Lo, p. 25] or [MQ1, Lemma 3.1]) that the central lattice can be described as

$$
\Gamma_{Z}(P, o)=\left\{X \in \mathfrak{a}: \alpha(X) \in \pi \mathbb{Z} \text { for all } \alpha \in \Omega_{P}\right\} .
$$

Let us further choose a fundamental Weyl chamber $\mathfrak{a}^{+} \subset \mathfrak{a}$ and denote by $\Sigma=\left\{\alpha_{1}, \ldots, \alpha_{r}\right\}$ the corresponding set of positive simple roots (see the appendix below); then

$$
\Gamma_{Z}(P, o)=\operatorname{span}_{\pi \mathbb{Z}}\left(\Sigma^{*}\right)=\left\{\sum_{j=1}^{r} \lambda_{j} \alpha_{j}^{*}: \lambda_{j} \in \pi \mathbb{Z}\right\},
$$

where $\Sigma^{*}=\left\{\alpha_{1}^{*}, \ldots, \alpha_{r}^{*}\right\}$ denotes the basis of $\mathfrak{a}$ which is dual to $\Sigma$.

Since any pole is an element of the center, the set $\mathcal{P}(P, o)$ of all poles of $(P, o)$ is a subset of $Z(P, o)$, and the pole lattice

$$
\Gamma_{\mathcal{P}}(P, o):=\{X \in \mathfrak{a}: \exp (X) . o \in \mathcal{P}(P, o)\}
$$


is an affine sublattice of $\Gamma_{Z}(P, o)$ (not containing zero). In view of Observation 9 , the pole lattice can also be described as

$$
\begin{aligned}
\Gamma_{\mathcal{P}}(P, o) & =\{X \in \mathfrak{a}: \exp (X) . o \in Z(P, o) \backslash\{o\}, \exp (2 X) . o=o\} \\
& =\left(\Gamma_{Z}(P, o) \cap(1 / 2) \Gamma_{o}(P, o)\right) \backslash \Gamma_{o}(P, o),
\end{aligned}
$$

where

$$
\Gamma_{o}(P, o):=\{X \in \mathfrak{a}: \exp (X) . o=o\}
$$

is the unit lattice of $(P, o)$. To get a particularly easy description of the unit lattice of $P$, we assume that $P$ is a simply connected irreducible symmetric space of compact type. To make visible in our notation when we assume simple connectedness, we replace $P$ by $\tilde{P}$.

Notice that if $\tilde{P}$ is simply connected, the isotropy group $\mathrm{K}$ in the connected transvection group $\mathrm{G}$ of $\tilde{P}=\mathrm{G} / \mathrm{K}$ is also connected; that is, $\mathrm{K}=\mathrm{K}_{e}$. This follows from the short exact sequence of homotopy groups

$$
\{0\}=\pi_{1}(\tilde{P}) \rightarrow \pi_{0}(\mathrm{~K}) \rightarrow \pi_{0}(\mathrm{G})=\{0\} .
$$

Following Loos [Lo, pp. 25, 69, 77], the unit lattice $\Gamma_{o}(\tilde{P}, o)$ can be described in terms of the system $\check{\Omega}_{\tilde{P}}$ of inverse roots (see equation (9) in the appendix):

$$
\Gamma_{o}(\tilde{P}, o)=\operatorname{span}_{\pi \mathbb{Z}}\left\{\check{\Omega}_{\tilde{P}}\right\} .
$$

Since $\Omega_{\tilde{P}}$ is reduced, the same holds true for $\check{\Omega}_{\tilde{P}}$ (see, e.g., [Bo, p. 197, Remark 2]), and the set $\check{\Sigma}:=\left\{\check{\alpha}_{1}, \ldots, \check{\alpha}_{r}\right\}$ is a system of simple roots in $\check{\Omega}_{\tilde{P}}$ (see [Se, p. 32, Proposition 7]). Thus, we can write

$$
\Gamma_{o}(\tilde{P}, o)=\operatorname{span}_{\pi \mathbb{Z}}\{\check{\Sigma}\} .
$$

We denote the inverse of the Cartan matrix $C$ of $\Sigma$ (see the appendix) by

$$
C^{-1}=\left(c_{1}^{-1}, \ldots, c_{r}^{-1}\right),
$$

where $c_{j}^{-1} \in \mathbb{R}^{r}$ is the $j$ th column of $C^{-1}$. The expansion of the simple dual roots in the basis of simple inverses roots given in (10) in the appendix implies the following.

Lemma 10. The vector $\pi \alpha_{j}^{*}$ lies in $\Gamma_{o}(\tilde{P}, o)$ if and only if $c_{j}^{-1} \in \mathbb{Z}^{r}$.

By (1) we get the following.

Lemma 11. An element $\pi \sum_{j=1}^{r} x_{j} \check{\alpha}_{j}$ of $\Gamma_{Z}(\tilde{P}, o)$ lies also in $\Gamma_{\mathcal{P}}(\tilde{P}, o)$ if and only if all its coefficients $x_{j}$ lie in $((1 / 2) \mathbb{Z})^{r} \backslash \mathbb{Z}^{r}$. In particular, $\pi \alpha_{j}^{*}$ lies in $\Gamma_{\mathcal{P}}(\tilde{P}, o)$ if and only if $c_{j}^{-1} \in((1 / 2) \mathbb{Z})^{r} \backslash \mathbb{Z}^{r}$. 


\subsection{Classification of centrioles in terms of roots}

We are now ready to describe all centriole points; these are points lying in some centriole, in a pointed irreducible simply connected symmetric space $(\tilde{P}, o)$ of compact type up to the action of $\mathrm{K}=\mathrm{K}_{e}$.

Let $x$ be a centriole point, and let $\gamma$ be a shortest geodesic in $\tilde{P}$ between $\gamma(0)=0$ and $\gamma((1 / 2) \pi)=x$. There is an element $k \in \mathrm{K}$ such that the initial direction $X:=\operatorname{Ad}_{\mathrm{G}}(k) \dot{\gamma}(0)$ of the geodesic $k \cdot \gamma$ lies in the closure $\overline{\mathfrak{a}^{+}}$of our previously chosen fundamental Weyl chamber $\mathfrak{a}^{+} \subset \mathfrak{a}$. Hence, the expansion of $X$ in the basis $\Sigma^{*}$ has only nonnegative coefficients. The point $k . x$ may differ from $x$, but it still lies in the same centriole by Lemma 4 . Corollary 6 shows that the point $\pi X$ lies in the polar lattice $\Gamma_{\mathcal{P}}(\tilde{P}, o)$.

The property that $\gamma$, and hence also $k \cdot \gamma$, is shortest on the interval $[0,(1 / 2) \pi]$ means that the vector $(1 / 2) \pi X$ lies inside or on the tangent cut locus of $\tilde{P}$ in $\mathfrak{p} \cong T_{p} \tilde{P}$. According to Sakai [S2, p. 198], the intersection $\operatorname{Cut}_{\tilde{P}}\left(\overline{\mathfrak{a}^{+}}\right)$of the tangent cut locus of $\tilde{P}$ in $\mathfrak{p}$ with the closed fundamental Weyl chamber $\overline{\mathfrak{a}^{+}}$is

$$
\operatorname{Cut}_{\tilde{P}}\left(\overline{\mathfrak{a}^{+}}\right)=\left\{Y \in \overline{\mathfrak{a}^{+}}: \delta(Y)=\pi\right\}
$$

where

$$
\delta=\sum_{j=1}^{r} h_{j} \alpha_{j}
$$

is the highest root corresponding to $\Sigma$. From the appendix, we have that all coefficients $h_{j}$ are strictly positive integers.

To sum up: to describe all centriole points of $(\tilde{P}, o)$ up to the action of $\mathrm{K}$, we have to look at the elements $X \in \overline{\mathfrak{a}^{+}}$that satisfy the two conditions:

$$
\begin{aligned}
\pi X & \in \Gamma_{\mathcal{P}}(\tilde{P}, o), \\
\frac{1}{2} \pi \delta(X) & \leq \pi \quad \text { or, equivalently, } \quad \delta(X) \leq 2 .
\end{aligned}
$$

TheOREM 12. There are four possible types of elements $X \in \overline{\mathfrak{a}^{+}}$that satisfy the conditions given in (3) and (4):

type I, $X=\alpha_{j}^{*}$, where $h_{j}=1$ and $c_{j}^{-1} \in((1 / 2) \mathbb{Z})^{r} \backslash \mathbb{Z}^{r}$; the vector $X$ is extrinsically symmetric, and $(1 / 2) \pi X$ lies in $(1 / 2) \operatorname{Cut}_{\tilde{P}}\left(\overline{\mathfrak{a}^{+}}\right)$;

type II, $X=\alpha_{j}^{*}$, where $h_{j}=2$ and $c_{j}^{-1} \in((1 / 2) \mathbb{Z})^{r} \backslash \mathbb{Z}^{r} ;$ the vector $(1 / 2) \pi X$ lies in $\operatorname{Cut}_{\tilde{P}}\left(\overline{\mathfrak{a}^{+}}\right)$; 
type III, $X=2 \alpha_{j}^{*}$, where $h_{j}=1$ and $c_{j}^{-1} \in((1 / 4) \mathbb{Z})^{r} \backslash((1 / 2) \mathbb{Z})^{r} ;$ the vector $\alpha_{j}^{*}$ is extrinsically symmetric, and $(1 / 2) \pi X$ lies in $\operatorname{Cut}_{\tilde{P}}\left(\overline{\mathfrak{a}^{+}}\right)$;

type IV $, X=\alpha_{j}^{*}+\alpha_{k}^{*}, k \neq j$, where $h_{j}=h_{k}=1$ and $c_{j}^{-1}+c_{k}^{-1} \in((1 / 2) \mathbb{Z})^{r} \backslash$ $\mathbb{Z}^{r}$; the vector $(1 / 2) \pi X$ lies in $\operatorname{Cut}_{\tilde{P}}\left(\overline{\mathfrak{a}^{+}}\right)$.

Conversely, any element $X$ of type I, II, III, or IV satisfies the requirements given in (3) and (4).

Proof. Any element $X \in \overline{\mathfrak{a}^{+}}$can be expanded in the basis of dual simple roots, $X=\sum_{j} x_{j} \alpha_{j}^{*}$, with nonnegative coefficients; that is, $x_{j} \geq 0$. Since $\pi X \in \Gamma_{\mathcal{P}}(\tilde{P}, o) \subset \Gamma_{Z}(\tilde{P}, o)$, the coefficients $x_{j}$ are, moreover, integers. We conclude that

$$
x_{j} \in \mathbb{N}=\{0,1,2, \ldots\} .
$$

Since $0 \notin \Gamma_{\mathcal{P}}(\tilde{P})$, at least one coefficient $x_{j}$ does not vanish. With $\delta=$ $\sum_{j=1}^{r} h_{j} \alpha_{j}$, (4) reads as follows:

$$
\sum_{j=1}^{r} h_{j} x_{j} \leq 2
$$

We distinguish several cases.

(1) Exactly one coefficient $x_{j}$ does not vanish.

(a) If $h_{j}=1$, there are two cases.

(i) Case $x_{j}=1$; then $X=\alpha_{j}^{*}$. Since $\pi X \in \Gamma_{\mathcal{P}}(\tilde{P}, o)$, equation (10) in the appendix and Lemma 11 show that $X$ is of type $\mathrm{I}$.

(ii) Case $x_{j}=2$; then $X=2 \alpha_{j}^{*}$. Since $\pi X \in \Gamma_{\mathcal{P}}(\tilde{P}, o)$, (10) and Lemma 11 show that $X=2 \alpha_{j}^{*}$ is of type III.

(b) If $h_{j}=2$, the only possibility is $x_{j}=1$, and by (10) and Lemma 11, $X$ is of type II, because $\pi X \in \Gamma_{\mathcal{P}}(\tilde{P}, o)$.

(2) Exactly two coefficients $x_{j}$ and $x_{k}(j \neq k)$ do not vanish. Since $h_{j} x_{j}$ and $h_{k} x_{k}$ are both greater than or equal to 1 and since $h_{j} x_{j}+h_{k} x_{k} \leq 2$, we get $h_{j}=x_{j}=h_{k}=x_{k}=1$, so that $X=\alpha_{j}^{*}+\alpha_{k}^{*}$. Equation (10) in the appendix yields $X=\alpha_{j}^{*}+\alpha_{k}^{*}=\sum_{l=1}^{r}\left(s_{j l}^{*}+s_{k l}^{*}\right) \check{\alpha}_{l}$, where $s_{j k}^{*}$ is the entry of $\left(C^{-1}\right)^{T}$ at position $(j, k)$. Lemma 11 shows that $X$ is of type IV.

(3) At least three coefficients $x_{j}, x_{k}$, and $x_{l}$ do not vanish. Since $h_{j} x_{j}, h_{k} x_{k}$, and $h_{l} x_{l}$ are all at least 1 , we get $h_{j} x_{j}+h_{k} x_{k}+h_{l} x_{l} \geq 3$. This contradicts equation (5). 
REMARK 13. Burns and Nagano discovered necessary conditions (which also involve roots) for a vector to be the initial direction of a shortest geodesic arc joining a base point to some polar (see [Bu3, Lemma 2.1, Proposition 2.2], [N1, Proposition 6.5, p. 72], [N2, p. 52], and [Bu2, Lemma 2.1, Proposition 2.2]). Their conditions and their proofs have some similarity to our conditions and our proof of Theorem 12. This is not astonishing. Indeed, the method used in these proofs shares common aspects with Loos's method to prove the classification of inner involutions of a compact simple Lie group (see [Lo, p. 121]), which may go back to Borel and de Siebenthal [BS]. Notice that in Theorem 12 only geodesics whose initial direction is of type I are shortest up to the pole.

Our next goal is to show that the centrioles corresponding to two different elements $X, Y \in \overline{\mathfrak{a}^{+}}$satisfying the conditions of equations (3) and (4) are distinct.

We first notice that two different elements $X, Y \in \overline{\mathfrak{a}^{+}}$are not conjugate by an element of $\mathrm{K}$, because every $\mathrm{K}$-orbit of the linear isotropy representation intersects a closed Weyl chamber in exactly one point (see [He, Chapter VII, Proposition 2.2, Theorem 2.22]).

Theorem 12 shows that at a first glance we can distinguish two kinds of centrioles.

(1) The centrioles of $(\tilde{P}, o)$ that do not intersect the cut locus of $(\tilde{P}, o)$ are formed by the midpoints of shortest geodesic arcs between $o$ and a pole of $(\tilde{P}, o)$. We call them s-centrioles or centrioles of s-type (compare also the notion of s-size in $[\mathrm{Tn}])$. S-centrioles are of the form $\mathrm{K} .(\exp ((1 / 2) \pi X) . o)$, where $X$ is an element of type I.

(2) The centrioles that are subsets of the cut locus of $(\tilde{P}, o)$ correspond to elements of type II, III, or IV in Theorem 12.

We see the following directly.

LEMMA 14. If $X \in \overline{\mathfrak{a}^{+}}$is an element of type $I$ and if $Y \in \overline{\mathfrak{a}^{+}}$is an element of type II, III, or IV, then the corresponding centrioles $\mathrm{K} .(\exp ((1 / 2) \pi X) . o)$ and $\mathrm{K} .(\exp ((1 / 2) \pi Y) . o)$ are disjoint.

LEMMA 15. If $X$ and $Y$ are two different elements of $\overline{\mathfrak{a}^{+}}$of type $I$, then the corresponding centrioles K. $(\exp ((1 / 2) \pi X) . o)$ and K. $(\exp ((1 / 2) \pi Y) . o)$ are disjoint.

Proof. In [MQ1, Lemma 4.4], the authors show that the poles $\exp (\pi X) . o$ and $\exp (\pi Y) . o$ of $(\tilde{P}, o)$ are different. The claim follows from Corollary 6 . 
REMARK 16. Both [MQ1, Lemma 4.4] and our Theorem 12 imply that the number of poles of $(\tilde{P}, o)$ coincides with the number of different elements of type I in the closed fundamental Weyl chamber $\overline{\mathfrak{a}^{+}}$. Since the description of these elements depends only on the root system of $\tilde{P}$ and not on the multiplicities, the number of poles of $(\tilde{P}, o)$ coincides with the number of poles of $(\tilde{\mathrm{H}}, e)$, where $\tilde{\mathrm{H}}$ is the connected simply connected compact simple Lie group whose root system is isomorphic to the one of $\tilde{P}$. One can verify that a pole of $(\tilde{\mathrm{H}}, e)$ is precisely an element of the (group-theoretic) center of $\tilde{H}$ that squares to the identity.

This implies that if the center of $(\tilde{P}, o)$ contains only one other point besides $o$ (which happens for the root systems of type $\mathfrak{b}_{r}, \mathfrak{c}_{r}$, and $\mathfrak{e}_{7}$; see [He, p. 516, Table IV]), then $(\tilde{P}, o)$ admits precisely one pole. The reason is that in this case the center of $\tilde{H}$ is isomorphic to $\mathbb{Z}_{2}$. If the root system of $(\tilde{P}, o)$ is of type $\mathfrak{e}_{6}$, then $(\tilde{P}, o)$ does not admit any pole.

We further observe that the center of most simply connected simple real Lie groups contains either no or just one element of order 2. The only exception is $\operatorname{Spin}_{4 n}(n \geq 2)$, whose center is isomorphic to $\mathbb{Z}_{2} \times \mathbb{Z}_{2}$ and hence contains three elements of order 2 . Thus, poles of $(\tilde{P}, o)$ are unique in most cases. The only exceptions are the spaces $\tilde{P}$ whose the root system is of type $\mathfrak{d}_{2 n}$ with $n \geq 2$, namely, $\tilde{P}=\operatorname{Spin}_{4 n}$ and $\tilde{P}=\tilde{G}_{2 n}\left(\mathbb{R}^{4 n}\right)=\mathrm{SO}_{4 n} /\left(\mathrm{SO}_{2 n} \times\right.$ $\mathrm{SO}_{2 n}$ ) with $n \geq 2$. These spaces, when pointed, admit three poles (see also [CN2, p. 293] and [N1, Section 2]).

We are left with the question of whether centrioles corresponding to different elements of type II, III, and IV are disjoint. Since these centrioles are subsets of the cut locus, our main tool is Sakai's description of the structure of the tangent cut locus of $(\tilde{P}, o)$. Following Sakai [S1], [S2] we define for

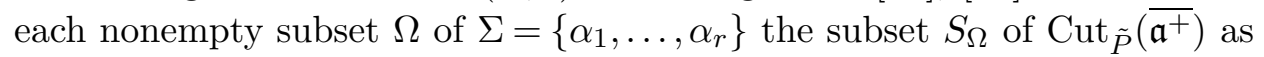
the set of all $X \in \operatorname{Cut}_{\tilde{P}}\left(\overline{\mathfrak{a}^{+}}\right)$satisfying the two conditions

$$
\begin{array}{ll}
\alpha(X)>0 & \text { if } \alpha \in \Omega, \\
\alpha(X)=0 & \text { if } \alpha \in \Sigma \backslash \Omega
\end{array}
$$

that is,

$$
S_{\Omega}=\left\{X \in \operatorname{Cut}_{\tilde{P}}\left(\overline{\mathfrak{a}^{+}}\right): \alpha(X)>0 \forall \alpha \in \Omega, \alpha(X)=0 \forall \alpha \in \Sigma \backslash \Omega\right\}
$$

LEMMA 17. We have the following. 
(1) If the coefficient $h_{j}$ of $\alpha_{j}$ in the highest root $\delta$ is $h_{j}=1$, then $S_{\left\{\alpha_{j}\right\}}=$ $\left\{\pi \alpha_{j}^{*}\right\}$.

(2) If the coefficient $h_{j}$ of $\alpha_{j}$ in the highest root $\delta$ is $h_{j}=2$, then $S_{\left\{\alpha_{j}\right\}}=$ $\left\{(1 / 2) \pi \alpha_{j}^{*}\right\}$.

(3) If $j \neq k$ and if the coefficients of $\alpha_{j}$ and $\alpha_{k}$ in the highest root are $h_{j}=h_{k}=1$, then

$$
S_{\left\{\alpha_{j}, \alpha_{k}\right\}}=\left\{x_{j} \alpha_{j}^{*}+x_{k} \alpha_{k}^{*}: x_{j}>0, x_{k}>0, x_{j}+x_{k}=\pi\right\} .
$$

In particular, $(1 / 2) \pi\left(\alpha_{j}^{*}+\alpha_{k}^{*}\right) \in S_{\left\{\alpha_{j}, \alpha_{k}\right\}}$.

Proof. Let $X=\sum_{l=1}^{r} x_{l} \alpha_{l}^{*}$ be an element of $\operatorname{Cut}_{\tilde{P}}\left(\overline{\mathfrak{a}^{+}}\right)$; that is, the coefficients $x_{l}$ are all nonnegative. Moreover, $\delta(X)=\pi$ by equation (2).

To show claim (1), assume that $X$ is an element of $S_{\left\{\alpha_{j}\right\}}$. By equation (6), we have $\alpha_{j}(X)=x_{j}>0$. From equation (7), we obtain $x_{l}=\alpha_{l}(X)=0$ if $l \neq j$. Finally, as $h_{j}=1$, we get $\delta(X)=x_{j}=\pi$. Thus, $X=\pi \alpha_{j}^{*}$.

The proof of claim (2) is similar. Assume that $X \in S_{\left\{\alpha_{j}\right\}}$. By equation (6), we have $x_{j}=\alpha_{j}(X)>0$. Using equation (7), we get $x_{l}=\alpha_{l}(X)=0$ if $l \neq j$. Finally, from $\delta(X)=\pi$ we conclude that $2 x_{j}=\pi$, because $h_{j}=2$. Therefore, $X=(1 / 2) \pi \alpha_{j}^{*}$.

To show claim (3), let $X \in S_{\left\{\alpha_{j}, \alpha_{k}\right\}}$. Equation (6) yields $x_{j}, x_{k}>0$, and by equation (7), $x_{l}=0$ if $l \neq j, k$. With $h_{j}=h_{k}=1$, we get $\delta(X)=x_{j}+x_{k}=\pi$.

Proposition 18 ([S2, Lemmas 4(2), 5(1)]).

(1) For any $\Omega \subset \Sigma$, the set $I_{\Omega}:=\left\{k .(\exp (X) . o): k \in \mathrm{K}, X \in S_{\Omega}\right\}$ is an embedded submanifold of $\tilde{P}$.

(2) We have $I_{\Omega} \cap I_{\Omega^{\prime}} \neq \emptyset$ if and only if $\Omega=\Omega^{\prime}$.

The proof of Proposition 18(1) can be found in [S1, proof of Proposition 4.10(iv)]. For the proof of Proposition 18(2), we refer to [S1, proof of Lemma 5.1]. Lemma 17 and Proposition 18 imply the following.

Lemma 19. Let $X, Y \in \overline{\mathfrak{a}^{+}}$be two different elements of type II, III, or $I V$. (The types of $X$ and $Y$ need not be different.) Then the corresponding centrioles K. $(\exp ((1 / 2) \pi X) . o)$ and K. $(\exp ((1 / 2) \pi Y) . o)$ are disjoint.

We summarize Lemmas 14, 15, and 19 in the following theorem.

THEOREM 20. Let $X$ and $Y$ be two different elements of $\overline{\mathfrak{a}^{+}}$satisfying the conditions given in equations (3) and (4). Then the centriole that contains $\exp ((1 / 2) \pi X)$.o is different from the centriole that contains $\exp ((1 / 2) \pi Y)$.o. 
REMARK 21. There may well be an isometry $g$ of $\tilde{P}$ fixing $o$ that maps $\exp ((1 / 2) \pi X) . o$ onto $\exp ((1 / 2) \pi Y) . o$, where $X$ and $Y$ are as in the assumptions of Theorem 20. But such an isometry $g$ is never a transvection.

Take, for example, $\tilde{P}=\operatorname{Spin}_{4 n}, n \geq 3$, endowed with the bi-invariant metric given by the Cartan-Killing form and the identity as a base point. The nontrivial Dynkin diagram automorphism of $\mathfrak{d}_{2 n}$ induces an isometry $g$ of $\tilde{P}$ that interchanges two extrinsically symmetric elements in the chosen closed fundamental Weyl chamber, which are not in the same component of the isotropy orbit.

REMARK 22. Theorems 12 and 20 show that the centrioles of a simply connected pointed symmetric space $(\tilde{P}, o)$ can be read off from its root system.

REMARK 23. In Lemma 1 we have seen that one can associate to a pole $p$ of $(\tilde{P}, o)$ a double covering $\pi_{p}: \tilde{P} \rightarrow \tilde{P} / \Gamma_{p}$ between symmetric spaces. Any centriole in the centrosome $C(o, p)$ of $(\tilde{P}, o)$ projects to a polar of $\left(\tilde{P} / \Gamma_{p}, \pi_{p}(o)\right)$. A classification list of polars can be found in [CN1] and [CN2]; a more detailed case-by-case determination of polars is described in [N1], and further proofs can be found in [N2]. Using the classification of polars, it is possible to establish case by case a list of all centrioles of $(\tilde{P}, o)$ lying in $C(o, p)$ by looking at those polars of $\left(\tilde{P} / \Gamma_{z}, \pi_{z}(o)\right)$ that are not projections of polars of $(\tilde{P}, o)$ (see also [Bu3] or [NS, 1.3b]).

We are not aware that a complete description of all shortest geodesics to centrioles in an irreducible simply connected pointed symmetric space of compact type in terms of its root system has been given thus far.

\subsection{Examples for each type}

In this section we present examples for all four types of elements mentioned in Theorem 12.

ExAmple 24 (type I). For any pole $p$ in an irreducible pointed symmetric space $(P, o)$ of compact type ( $P$ need not be simply connected $)$, there exists at least one centriole that consists of midpoints of shortest geodesic arcs in $P$ joining $o$ to $p$. These s-centrioles correspond to extrinsic symmetric tangent vectors (see [MQ1]). Extrinsic symmetric vectors in a closed Weyl chamber are precisely the dual vectors of simple roots whose coefficient in the highest root is 1 (see, e.g., [MQ1, Lemma 2.1] and [KN]). Easy examples are equatorial hyperspheres in spheres. 
If the simply connected irreducible compact symmetric space $\tilde{P}$ has Dynkin type $\mathfrak{c}_{r}, r \geq 2$, then $(\tilde{P}, o)$ has only one centriole (see [N2, Proposition $2.23(\mathrm{i})])$ which is a fortiori an s-centriole. These spaces $\tilde{P}$ include irreducible Hermitian symmetric spaces of compact type whose noncompact dual spaces can be realized as tube domains (are of tube type), such as $\mathrm{E}_{7} /\left(S^{1} \mathrm{E}_{6}\right)$.

Example 25 (type II). The Cartan matrix of the Dynkin diagram

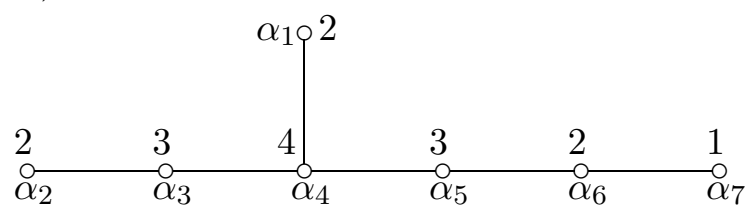

of type $\mathfrak{e}_{7}$ (with each root labeled with its coefficient in the highest root; compare [He, p. 477]) is

$$
C=\left(\begin{array}{ccccccc}
2 & 0 & 0 & -1 & 0 & 0 & 0 \\
0 & 2 & -1 & 0 & 0 & 0 & 0 \\
0 & -1 & 2 & -1 & 0 & 0 & 0 \\
-1 & 0 & -1 & 2 & -1 & 0 & 0 \\
0 & 0 & 0 & -1 & 2 & -1 & 0 \\
0 & 0 & 0 & 0 & -1 & 2 & -1 \\
0 & 0 & 0 & 0 & 0 & -1 & 2
\end{array}\right)
$$

(compare [He, p. 474]), and its inverse is

$$
C^{-1}=\left(\begin{array}{ccccccc}
\frac{7}{2} & 2 & 4 & 6 & \frac{9}{2} & 3 & \frac{3}{2} \\
2 & 2 & 3 & 4 & 3 & 2 & 1 \\
4 & 3 & 6 & 8 & 6 & 4 & 2 \\
6 & 4 & 8 & 12 & 9 & 6 & 3 \\
\frac{9}{2} & 3 & 6 & 9 & \frac{15}{2} & 5 & \frac{5}{2} \\
3 & 2 & 4 & 6 & 5 & 4 & 2 \\
\frac{3}{2} & 1 & 2 & 3 & \frac{5}{2} & 2 & \frac{3}{2}
\end{array}\right)
$$

By $\mathrm{E}_{7}$ we denote the simply connected and connected compact real Lie group whose root system is of type $\mathfrak{e}_{7}$. Theorems 12 and 20 imply the following.

The simply connected irreducible pointed compact symmetric spaces $(\tilde{P}, o)$ where the root system of $\tilde{P}$ is of type $\mathfrak{e}_{7}$ (which are $\tilde{P}=\mathrm{E}_{7}$ and $\left.\tilde{P}=\mathrm{E}_{7} / \mathrm{SU}_{8}\right)$ have two centrioles:

- an s-centriole defined by $\alpha_{7}^{*}$; 
- a centriole defined by $\alpha_{1}^{*}$, which is of type II.

(See also [NS, proof of Proposition 4.10] and [N2].)

Example 26 (type III). If $X=2 \alpha_{j}^{*}$ is of type III, then [MQ1, Proposition 4.2] shows that the corresponding centriole is a singleton formed by an element of the center of $(\tilde{P}, o)$. Examples of such center elements occur in $\tilde{P}=\mathrm{SU}_{4 n}$. Let us explain this in the easiest case $\tilde{P}=\mathrm{SU}_{4}$. The root system of $\mathrm{SU}_{4}$ has type $\mathfrak{a}_{3}$, and every simple root has coefficient 1 in the highest root (see, e.g., [He, p. 477]). Using the enumeration and notation of [He, Chapter X, Section 3], the Cartan matrix and its inverse are

$$
C=\left(\begin{array}{ccc}
2 & -1 & 0 \\
-1 & 2 & -1 \\
0 & -1 & 2
\end{array}\right) \quad \text { and } \quad C^{-1}=\left(\begin{array}{ccc}
\frac{3}{4} & \frac{1}{2} & \frac{1}{4} \\
\frac{1}{2} & 1 & \frac{1}{2} \\
\frac{1}{4} & \frac{1}{2} & \frac{3}{4}
\end{array}\right)
$$

This shows that $2 \alpha_{1}^{*}$ and $2 \alpha_{3}^{*}$ are elements of type III.

ExAmple 27 (type IV). To find an example of elements of type IV, we consider a root system of type $\mathfrak{d}_{4}$, for example, $\tilde{P}=\operatorname{Spin}_{8}$. The corresponding Dynkin diagram labeled with its coefficients in the highest root is (see, e.g., [He, p. 477])

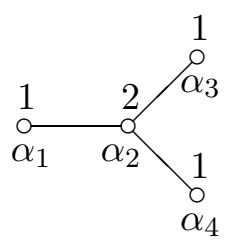

The roots $\alpha_{1}, \alpha_{3}$, and $\alpha_{4}$ have coefficient 1 in the highest root. The Cartan matrix $C$ (see, e.g., [He, p. 464]) and its inverse are

$$
C=\left(\begin{array}{cccc}
2 & -1 & 0 & 0 \\
-1 & 2 & -1 & -1 \\
0 & -1 & 2 & 0 \\
0 & -1 & 0 & 2
\end{array}\right) \quad \text { and } \quad C^{-1}=\left(\begin{array}{cccc}
1 & 1 & \frac{1}{2} & \frac{1}{2} \\
1 & 2 & 1 & 1 \\
\frac{1}{2} & 1 & 1 & \frac{1}{2} \\
\frac{1}{2} & 1 & \frac{1}{2} & 1
\end{array}\right)
$$

This shows that $\alpha_{1}^{*}+\alpha_{3}^{*}, \alpha_{1}^{*}+\alpha_{4}^{*}$, and $\alpha_{3}^{*}+\alpha_{4}^{*}$ are of type IV.

\section{$\S 3$. S-centrioles}

\subsection{S-centrioles as embedded $R$-spaces}

Whenever an irreducible pointed symmetric space $(P, o)$ of compact type, which is not necessarily simply connected, admits a pole $p$, then $p$ lies in 
the center of $(P, o)$. The set of all midpoints of shortest geodesics between $o$ and $p$ decomposes into $\mathrm{K}_{e}$-orbits (see [MQ1, Theorem 1.2]). Each of these s-centrioles is an isometric embedding of an extrinsic symmetric space in $\mathfrak{p}$ (see [MQ1, Theorem 1.3(a)]) or, in other words, an embedded symmetric $R$-space. Thus, we can state the following.

THEOREM 28. Every irreducible pointed symmetric space of compact type that admits a pole contains a centriole that is an embedded symmetric $R$-space.

One should compare this result with [NT1, Corollary 5.10, Theorem 5.11(i)] and [NT2, p. 414].

\subsection{S-centrioles as maximal totally geodesic submanifolds}

We call a proper complete totally geodesic submanifold $M \subset P, M \neq P$, maximal if $M$ is not contained in any other complete totally geodesic submanifold of $P$ except $M$ and $P$ themselves. Surely equatorial hyperspheres in spheres are maximal totally geodesic submanifolds. On the other hand, the singleton centrioles from Example 26 show that we cannot expect that any centriole is a maximal totally geodesic submanifold. But it turns out that several centrioles, especially all s-centrioles, are maximal totally geodesic submanifolds.

Since s-centrioles are isometric embeddings of extrinsically symmetric $s$-orbits in $\mathfrak{p}$ (see [MQ1]), it is necessary to present some facts on these orbits (see also [F1], [F2], [EH], and [BCO, Chapter 3]).

Let $(P, o)$ be an irreducible pointed symmetric space of compact type, let $\mathfrak{g}=\mathfrak{k} \oplus \mathfrak{p}$ be the corresponding Cartan decomposition of its transvection Lie algebra $\mathfrak{g}$, and let $\left(\sigma_{o}\right)_{*}$ be the corresponding involution of $\mathfrak{g}$ (see the appendix). Up to a positive factor, the Cartan-Killing form of $\mathfrak{g}$ induces the Riemannian metric on $\mathfrak{p} \cong T_{o} P$. Let $\xi \neq 0$ be an extrinsically symmetric element in $\mathfrak{p}$, that is, $\operatorname{ad}(\xi)^{3}=-\operatorname{ad}(\xi)$; or, equivalently, the spectrum of $\operatorname{ad}(\xi)$ consists only of the eigenvalues $\pm i$ and 0 . The isotropy orbit $M:=$ $\operatorname{Ad}_{\mathrm{G}}\left(\mathrm{K}_{e}\right) \xi \subset \mathfrak{p}$ of $\xi$ carries the natural transitive isometric $\mathrm{K}_{e}$-action

$$
\mathrm{K}_{e} \times M \rightarrow M, \quad(k, m) \mapsto \operatorname{Ad}_{\mathrm{G}}(k) m .
$$

$M$ is known to be an extrinsically symmetric submanifold of $\mathfrak{p}$; that is, $M$ is invariant under the reflections through all its normal spaces.

Since $\xi \in \mathfrak{p}$, and since $\operatorname{Ad}(\exp (\pi \xi))=\operatorname{Ad}(\exp (-\pi \xi))$, the involutive automorphism $\tau:=\operatorname{Ad}(\exp (\pi \xi))=e^{\pi \operatorname{ad}(\xi)}$ commutes with $\left(\sigma_{o}\right)_{*}$. Thus, we have 
two orthogonal splittings (with respect to the Cartan-Killing form of $\mathfrak{g}$ )

$$
\mathfrak{k}=\mathfrak{k}_{+} \oplus \mathfrak{k}_{-} \quad \text { and } \quad \mathfrak{p}=\mathfrak{p}_{+} \oplus \mathfrak{p}_{-},
$$

where $\tau$ is the identity on $\mathfrak{k}_{+} \oplus \mathfrak{p}_{+}$, and $\mathfrak{k}_{-} \oplus \mathfrak{p}_{-}$is the $(-1)$-eigenspace of $\tau$. The linear space $\mathfrak{p}_{+}=\{X \in \mathfrak{p}: \operatorname{ad}(\xi) X=0\}$ is the normal space of $N_{\xi} M$. Therefore, $\mathfrak{p}_{-}$is the tangent space $T_{\xi} M$. This shows that $\left.\tau\right|_{\mathfrak{p}}$ is the extrinsic symmetry of $M$ at $\xi$.

We want to show that $\mathfrak{k}$ is the Lie algebra of infinitesimal transvections of $M$. Since $P$ is an irreducible symmetric space, the isotropy action of $\mathrm{K}_{e}$ on $\mathfrak{p}$ is irreducible, too. It follows that $M$ is a full submanifold of $\mathfrak{p}$, that is, that $M$ is not contained in any proper affine linear subspace of $\mathfrak{p}$. Therefore, an element $k \in \mathrm{K}_{e}$ that acts trivially on $M$ acts as the identity on $\mathfrak{p} \cong T_{o} P$ and therefore on $P$, too. This shows that the action of $\mathrm{K}_{e}$ on $M$ is effective. We therefore consider $\mathrm{K}_{e}$ as a subgroup of the isometry group of $M$.

The subspace $\mathfrak{k}_{-} \subset \mathfrak{k}$ is a Lie triple corresponding to the symmetric space $M$. It can be identified with $T_{\xi} M$ by the differential of the principal bundle $\mathrm{K}_{e} \rightarrow M, k \mapsto \operatorname{Ad}_{\mathrm{G}}(k) \xi$ at the identity, which coincides with $-\operatorname{ad}(\xi)$. The transvection Lie algebra $\mathfrak{h}$ of $M$ is therefore generated by $\mathfrak{k}_{-}$; that is, $\mathfrak{h}:=$ $\left[\mathfrak{k}_{-}, \mathfrak{k}_{-}\right] \oplus \mathfrak{k}_{-} \subseteq \mathfrak{k}$.

Let $\mathfrak{h}^{\perp}$ be the orthogonal complement (with respect to the Cartan-Killing form $\kappa$ of $\mathfrak{g}$ ) of $\mathfrak{h}$ in $\mathfrak{k}$. Then $\mathfrak{h}^{\perp}$ is a subspace of the isotropy Lie algebra $\mathfrak{k}_{+}$ of $(M, \xi)$ in $\mathfrak{k}$. Take $Z \in \mathfrak{h}^{\perp}$. For all $X, Y \in \mathfrak{k}_{-}$, we have $[X, Y] \in \mathfrak{h}$. Hence, $\kappa([Z, X], Y)=\kappa(Z,[X, Y])=0$, and we conclude that $\operatorname{ad}(Z)$ vanishes on $\mathfrak{k}_{-}$. Hence, the 1 -parameter subgroup $t \mapsto \exp (t Z)$ acts trivially on $\mathfrak{k}_{-}$and therefore also trivially on $M$. As K acts effectively on $M$, we conclude that $Z$ vanishes; that is, $\mathfrak{h}^{\perp}=\{0\}$, or, in other words, $\mathfrak{h}=\mathfrak{k}$. This shows that $\mathfrak{k}$ is the transvection Lie algebra of $M$.

LEMMA 29. The Lie algebra of the transvection group of an s-centriole in an irreducible pointed symmetric space of compact type $(P, o)$ coincides with the Lie algebra $\mathfrak{k}$ of the isotropy group $\mathrm{K}$ of $(P, o)$.

Proof. Let $C_{x}(o, p)$ be an s-centriole. Then there is an extrinsically symmetric element $\xi$ in $\mathfrak{p}$ with the property that the map

$$
M:=\operatorname{Ad}_{\mathrm{G}}\left(K_{e}\right) \xi \rightarrow P, \quad X \mapsto \exp \left(\frac{\pi}{2} X\right) . o
$$

is a $\mathrm{K}_{e}$-equivariant isometric embedding whose image is just $C_{x}(o, p)$ (see [MQ1, Theorem 1.3(a)]). Since the transvection Lie algebra of $M$ coincides with $\mathfrak{k}$, the same holds true for $C_{x}(o, p)$. 
Recall that because $P$ is irreducible, $\mathfrak{k}$ is a maximal proper subalgebra of $\mathfrak{g}$; that is, the only Lie subalgebras of $\mathfrak{g}$ that contain $\mathfrak{k}$ are $\mathfrak{k}$ and $\mathfrak{g}$ themselves.

Indeed, let $\mathfrak{u}$ be a Lie subalgebra of $\mathfrak{g}$ that satisfies $\mathfrak{k} \subsetneq \mathfrak{u} \subsetneq \mathfrak{g}$. Since $\mathfrak{g}=$ $\mathfrak{k} \oplus \mathfrak{p}$ is an orthogonal splitting with respect to the Killing form, we can write $\mathfrak{u}=\mathfrak{k} \oplus \mathfrak{m}$ for a nonzero proper linear subspace $\mathfrak{m}=\mathfrak{u} \cap \mathfrak{p} \subset \mathfrak{p}$. But then $[\mathfrak{k}, \mathfrak{m}] \subset \mathfrak{m}$, contradicting the fact that $\operatorname{ad}_{\mathfrak{g}}(\mathfrak{k})$ is irreducible on $\mathfrak{p}$ (see $[\mathrm{He}$, p. 377]).

THEOREM 30. An s-centriole in an irreducible pointed symmetric space of compact type $(P, o)$ is a maximal totally geodesic submanifold.

Proof. Recall that the Lie algebra of infinitesimal transvections of any symmetric space $P$ is generated by any of its tangent Lie triples; more precisely, if $P$ is a symmetric space and if $\mathfrak{p}$ is a subspace of the Lie algebra of infinitesimal isometries of $P$ that is a tangent Lie triple of $P$, then the Lie algebra of the transvection group of $P$ is $[\mathfrak{p}, \mathfrak{p}] \oplus \mathfrak{p}$.

Let $C_{x}(o, p)$ be an s-centriole of $(P, o)$, and let $\mathfrak{c} \subset \mathfrak{k}$ be the tangent Lie triple of $C_{x}(o, p)$ that is identified with $T_{x} C_{x}(o, p)$. Lemma 29 yields $\mathfrak{k}=$ $[\mathfrak{c}, \mathfrak{c}] \oplus \mathfrak{c}$.

Assume that there exists a complete totally geodesic submanifold $M$ of $P$ with the property $C_{x}(o, p) \subsetneq M \subsetneq P$, and let $\mathfrak{m}$ be the tangent Lie triple of $M$ that is identified with $T_{x} M$. Then the transvection Lie algebra of $M$ is $[\mathfrak{m}, \mathfrak{m}] \oplus \mathfrak{m}$. We conclude that $\mathfrak{k} \subsetneq[\mathfrak{m}, \mathfrak{m}] \oplus \mathfrak{m} \subsetneq \mathfrak{g}$. This contradicts the irreducibility of $P$.

REMARK 31. Our proof of Theorem 30 still works for a lot of other positive-dimensional centrioles in an irreducible pointed symmetric space $(P, o)$, but there are some exceptions, too.

Since our proof of Theorem 30 relies on Lemma 29, we look for centrioles which are not of s-type and whose transvection Lie algebra is $\mathfrak{k}$.

Let $C_{x}(o, p)$ be such a centriole corresponding to some pole $p$ of $(P, o)$. The projection $\pi: P \rightarrow \operatorname{Ad}(P)$ identifies $o$ and $p$. Moreover, $\pi\left(C_{x}(o, p)\right)$ is a polar of $\operatorname{Ad}(P)$ that is locally isometric to $C_{x}(o, p)$.

If one looks at the classification of polars in irreducible adjoint spaces of Dynkin type $\mathfrak{a}_{r}, \mathfrak{b}_{r}, \mathfrak{c}_{r}, \mathfrak{d}_{r}$, and $\mathfrak{e}_{7}$ in [CN2, Appendix] and [N1, Sections 3, 4] one sees that Lemma 29 still holds for other positive-dimensional centrioles up to the following possible exceptions:

(1) the simply connected space $P=\mathrm{E}_{7} /\left(S^{1} \mathrm{E}_{6}\right)$;

(2) the simply connected Grassmannian $P=\tilde{G}_{r}\left(\mathbb{R}^{n}\right)$ of oriented $r$-dimensional real linear subspaces of $\mathbb{R}^{n}$ with $2 r \neq n$. 
$\mathrm{E}_{7} /\left(S^{1} \mathrm{E}_{6}\right)$ is a Hermitian symmetric space of Dynkin diagram type $\mathfrak{c}_{3}$ and therefore has only one centriole, which is of s-type (see [N2, Proposition 2.23(i)]).

Case (2) yields examples of positive-dimensional centrioles whose Lie algebra of infinitesimal transvections is not $\mathfrak{k}$. We present a first example, but similar phenomena also occur in some higher Grassmannians.

The simply connected Grassmannian $\tilde{G}_{3}\left(\mathbb{R}^{7}\right)$ has Dynkin type $\mathfrak{b}_{3}$ :

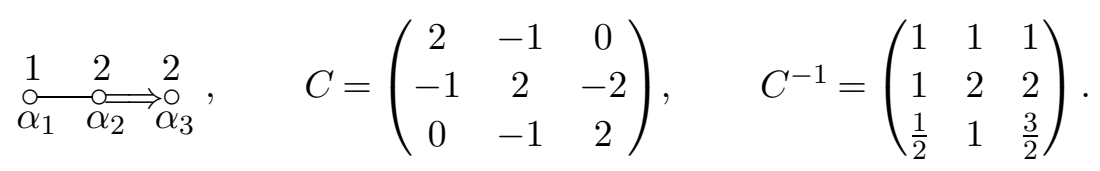

(The information for this Dynkin diagram labeled with coefficients in the highest root and the Cartan matrix above is taken from [He, pp. 463, 477].) According to Theorem 12, the element $X=\alpha_{3}^{*}$ is of type II and defines a centriole in $\tilde{G}_{3}\left(\mathbb{R}^{7}\right)$. Since Lemma 29 is a statement of infinitesimal nature, we can also look at the polar $P_{+}$in the (usual) Grassmannian $G_{3}\left(\mathbb{R}^{7}\right)$ that arises from the projection of the centriole mentioned before.

The standard subspace $\mathbb{R}^{3}=\operatorname{span}_{\mathbb{R}}\left(e_{1}, e_{2}, e_{3}\right) \subset \mathbb{R}^{7}$ serves as our base point $o \in G_{3}\left(\mathbb{R}^{7}\right)$. This yields

$$
\begin{aligned}
& \mathfrak{k}=\left\{\left(\begin{array}{cc}
A & 0 \\
0 & B
\end{array}\right): A \in \mathfrak{o}_{3}, B \in \mathfrak{o}_{4}\right\} \cong \mathfrak{o}_{3} \times \mathfrak{o}_{4} ; \\
& \mathfrak{p}=\left\{\left(\begin{array}{cc}
0 & Y \\
-Y^{T} & 0
\end{array}\right): Y \in \mathbb{R}^{3 \times 4}\right\}
\end{aligned}
$$

The maximal abelian subspace of $\mathfrak{p}$ of our choice is

$$
\mathfrak{a}=\left\{A^{\prime}=\left(\begin{array}{cc}
0 & A \\
-A^{T} & 0
\end{array}\right): A=\left(a_{j k}\right) \in \mathbb{R}^{3 \times 4}, \text { where } a_{j k}=0 \text { if } j \neq k\right\} .
$$

Following [He, p. 463], we take

$$
\alpha_{1}\left(A^{\prime}\right):=a_{11}-a_{22}, \quad \alpha_{2}\left(A^{\prime}\right):=a_{22}-a_{33}, \quad \text { and } \quad \alpha_{3}\left(A^{\prime}\right)=a_{33}
$$

as simple roots defining a positive fundamental Weyl chamber in $\mathfrak{a}$. We conclude that

$$
X:=\alpha_{3}^{*}=\left(\begin{array}{ccc}
0 & I_{3} & 0 \\
-I_{3} & 0 & 0 \\
0 & 0 & 0
\end{array}\right) \in \mathfrak{a}
$$


and hence

$$
\exp (t X)=\left(\begin{array}{ccc}
\cos (t) I_{3} & \sin (t) I_{3} & 0 \\
-\sin (t) I_{3} & \cos (t) I_{3} & 0 \\
0 & 0 & 1
\end{array}\right)
$$

and

$$
x=\exp \left(\frac{\pi}{2} X\right) \cdot o=\left(\begin{array}{ccc}
0 & I_{3} & 0 \\
-I_{3} & 0 & 0 \\
0 & 0 & 1
\end{array}\right) \cdot \operatorname{span}_{\mathbb{R}}\left(e_{1}, e_{2}, e_{3}\right)=\operatorname{span}_{\mathbb{R}}\left(e_{4}, e_{5}, e_{6}\right) .
$$

The centriole in $\tilde{G}_{3}\left(\mathbb{R}^{7}\right)$ that corresponds to $X$ covers a polar $P_{+}$in $G_{3}\left(\mathbb{R}^{7}\right)$. This polar $P_{+}$is the orbit of $x$ under the action of the group $\mathrm{S}\left(\mathrm{O}_{3} \times \mathrm{O}_{4}\right)$. Every point in $P_{+}$is a subspace of $\operatorname{span}_{\mathbb{R}}\left(e_{4}, e_{5}, e_{6}, e_{7}\right)$. Hence, the connected subgroup of $\mathrm{S}\left(\mathrm{O}_{3} \times \mathrm{O}_{4}\right)$ with Lie algebra $\mathfrak{o}_{3} \times\{0\}$ acts trivially on $P_{+}$.

\section{Appendix. Root systems}

The geometry of a simply connected pointed symmetric space of compact type can be encoded algebraically in a root system. To make this article more self-contained and to introduce the necessary terminology and notation, we give a brief overview of the theory of root systems of symmetric spaces needed in this article. Further details and proofs can be found in many standard references, such as [He], [Lo], [Bo], and [Se].

Let $(P, o)$ be an irreducible simply connected pointed symmetric space of compact type; that is, $P$ is compact and not a product of two symmetric spaces. As in Section 1.2, we denote by G its transvection group, which in this case is the identity component of its full isometry group, and we denote by $\mathrm{K}$ the G-stabilizer of the base point $o$. The Lie group $\mathrm{G}$ is compact and semisimple. Thus, the Cartan-Killing form

$$
\kappa(X, Y):=\operatorname{trace}(\operatorname{ad}(X) \circ \operatorname{ad}(Y)), \quad X, Y \in \mathfrak{g}
$$

of $\mathfrak{g}$ is negative definite.

The geodesic symmetry $s_{o}$ of $P$ at $o$ defines an involution

$$
\sigma_{o}: \mathrm{G} \rightarrow \mathrm{G}, \quad g \mapsto s_{o} g s_{o}
$$

Its derivative at the identity, $\left(\sigma_{o}\right)_{*}$, is an involution of the Lie algebra $\mathfrak{g}$ of $\mathrm{G}$. The fixed space $\mathfrak{k}$ of $\left(\sigma_{o}\right)_{*}$ is the Lie algebra of $\mathrm{K}$, and the $(-1)$-eigenspace of $\left(\sigma_{o}\right)_{*}$, denoted by $\mathfrak{p}$, can be identified with $T_{o} P$ by restricting the differential 
of the principal bundle $\mathrm{G} \rightarrow P, g \rightarrow g . o$ at the identity to $\mathfrak{p}$. We call $\mathfrak{p}$ a tangent Lie triple of $P$. Since $P$ is irreducible, the scalar product on $T_{o} P$ coincides up to a negative factor with the restriction of the Cartan-Killing $\kappa$ to $\mathfrak{p}$.

Every isometry $k \in \mathrm{K}$ acts on $T_{o} P$ by its derivative at $o$. The resulting representation of $\mathrm{K}$ is called linear isotropy representation. Under the above identification of $T_{o} P$ with $\mathfrak{p}$, this action becomes the restriction of $\operatorname{Ad}_{\mathrm{G}}(k)$ on $\mathfrak{p}$.

The curvature tensor on $T_{o} P \cong \mathfrak{p}$ can be written in terms of the Lie bracket as $R(X, Y) Z=-[[X, Y], Z]=-\operatorname{ad}(\operatorname{ad}(X) Y) Z$. Since the curvature tensor is a very important geometric quantity, we take a closer look at the derivations $\operatorname{ad}(X)$ of $\mathfrak{g}$ with $X \in \mathfrak{p}$. For this we choose a maximal abelian subspace $\mathfrak{a}$ of $\mathfrak{p}$. Any two maximal abelian subspaces of $\mathfrak{p}$ are conjugate under the isotropy action. Since $\{\operatorname{ad}(A): A \in \mathfrak{a}\}$ is a system of commuting skew-symmetric (with respect to the Cartan-Killing form) linear maps, its elements can be diagonalized simultaneously with purely imaginary eigenvalues. The set

$$
\Omega_{P}:=\left\{\alpha \in \mathfrak{a}^{*}: \alpha \neq 0, \mathfrak{g}_{\alpha} \neq\{0\}\right\},
$$

where $\mathfrak{a}^{*}$ is the set of all real-valued linear forms on $\mathfrak{a}$ and where $\mathfrak{g}_{\alpha}:=\{X \in$ $\mathfrak{g} \otimes \mathbb{C}: \operatorname{ad}(A) X=i \alpha(A) X$ for all $A \in \mathfrak{a}\}$, is called the root system of $P$, and its elements are called roots.

The Cartan-Killing form $\kappa$ induces a scalar product on $\Omega_{P}$ as follows. For each root $\alpha$, the root vector $H_{\alpha}$ is defined by $\alpha(A)=-\kappa\left(H_{\alpha}, A\right)$ for all $A \in \mathfrak{a}$. For two roots $\alpha$ and $\beta$, we define

$$
\langle\alpha, \beta\rangle:=-\kappa\left(H_{\alpha}, H_{\beta}\right)
$$

Since $P$ is irreducible, the same holds true for $\Omega_{P}$; that is, no root is perpendicular to all other roots.

The kernel of each root is a real hyperplane in $\mathfrak{a}$, and each connected component of

$$
\mathfrak{a} \backslash \bigcup_{\alpha \in \Omega_{P}} \operatorname{kernel}(\alpha)
$$

is a simplicial cone called a (fundamental) Weyl chamber. Any two Weyl chambers in $\mathfrak{p}$ are conjugate under the isotropy representation.

The root system $\Omega_{P}$ is called reduced if, for any root $\alpha \in \Omega_{P}$, the only multiples of $\alpha$ that are roots are precisely $\pm \alpha$. For our purposes (see Observation 7 ), we can restrict our attention to symmetric spaces $P$ whose root 
system is reduced. We now choose a fundamental Weyl chamber $\mathfrak{a}^{+}$and look at the set $\Sigma=\left\{\alpha_{1}, \ldots, \alpha_{r}\right\}$ formed by those roots $\alpha_{j}$ that satisfy

- $\alpha_{j}>0$ on $\mathfrak{a}^{+}$;

- $\operatorname{kernel}\left(\alpha_{j}\right)$ bounds $\mathfrak{a}^{+}$.

The system $\Sigma$ is a system of simple roots; that is,

- $r$ is the real dimension of $\mathfrak{a}$, called the rank of $P$;

- each root $\alpha$ can be written as a linear combination of elements of $\Sigma$ with either only nonnegative coefficients (positive roots) or only nonpositive coefficients (negative roots).

There is a highest root $\delta$ in $\Omega_{P}$ characterized by the fact that each coefficient of $\delta$ in the basis $\Sigma$ is not smaller than the corresponding coefficient of any other root in the basis $\Sigma$.

The geometry of $\Sigma$ can be encoded in the (invertible) Cartan matrix $C=\left(c_{j k}\right) \in \mathbb{R}^{r \times r}$ whose coefficients are

$$
c_{j k}=2 \frac{\left\langle\alpha_{j}, \alpha_{k}\right\rangle}{\left|\alpha_{k}\right|^{2}} .
$$

Finally, we denote by $\Sigma^{*}=\left\{\alpha_{1}^{*}, \ldots, \alpha_{r}^{*}\right\}$ the dual basis of $\Sigma$ defined by

$$
\alpha_{j}\left(\alpha_{k}^{*}\right)=\delta_{j k}:= \begin{cases}1, & j=k, \\ 0, & j \neq k .\end{cases}
$$

We can construct another root system from $\Omega_{P}$ as follows. Given $\alpha \in \Omega_{P}$, we define its inverse root by

$$
\check{\alpha}:=2 \frac{H_{\alpha}}{|\alpha|^{2}},
$$

and the set $\check{\Omega}_{P}$ of all inverse roots is called the inverse root system of $P$. It turns out (see, e.g., [Se, p. 32]) that $\check{\Sigma}=\left\{\check{\alpha}_{1}, \ldots, \check{\alpha}_{r}\right\}$ is a system of simple roots of $\check{\Omega}_{P}$ and therefore is a basis of $\mathfrak{a}$.

We now want to express the vectors of the dual basis $\Sigma^{*}$ in the basis $\check{\Sigma}$ of a. For this we first set $H_{\alpha_{j}}=\sum_{k=1}^{r} s_{j k} \alpha_{k}^{*}$ and get $\left\langle H_{\alpha_{l}}, H_{\alpha_{j}}\right\rangle=\alpha_{l}\left(H_{\alpha_{j}}\right)=$ $\sum_{k=1}^{r} s_{j k} \alpha_{l}\left(\alpha_{k}^{*}\right)=s_{j l}$. Since $\check{\alpha}_{j}=2\left(H_{\alpha_{j}} /\left|\alpha_{j}\right|^{2}\right)$, we obtain $\check{\alpha}_{j}=\sum_{k=1}^{r} \check{s}_{j k} \alpha_{k}^{*}$, where $\check{s}_{j k}=2\left(\left\langle H_{\alpha_{j}}, H_{\alpha_{k}}\right\rangle /\left|\alpha_{j}\right|^{2}\right)=: c_{k j}$ is a coefficient of the Cartan matrix (see equation $(8))$. We conclude that

$$
\alpha_{j}^{*}=\sum_{k=1}^{r} s_{j k}^{*} \check{\alpha}_{k},
$$


where $s_{j k}^{*}$ is the entry of the matrix $\left(C^{-1}\right)^{T}$ at position $(j, k)$. For an explicit formula for the entries of $C^{-1}$, we refer the reader to [LT].

Acknowledgments. The contents of Sections 1 and 2 are part of the author's habilitation dissertation [Q2]. The author wishes to express his gratitude to J.-H. Eschenburg, E. Heintze, and T. Vlachos for interesting discussions and hints.

\section{REFERENCES}

[BCO] J. Berndt, S. Console, and C. Olmos, Submanifolds and Holonomy, Chapman \& Hall/RC Res. Notes Math. 434, Chapman \& Hall, Boca Raton, Fla., 2003. MR 1990032. DOI 10.1201/9780203499153.

[BS] A. Borel and J. de Siebenthal, Les sous-groupes fermés de rang maximum des groupes de Lie clos, Comment. Math. Helv. 23 (1949), 200-221. MR 0032659.

[Bt] R. Bott, The stable homotopy of the classical groups, Ann. of Math. (2) 70 (1959), 313-337. MR 0110104.

[Bo] N. Bourbaki, Éléments de mathématique, fasc. 34: Groupes et algèbres de Lie, chapitres 4-6, Masson, Paris, 1981. MR 0647314.

[Bu1] J. M. Burns, Homotopy of compact symmetric spaces, Glasg. Math. J. 34 (1992), 221-228. MR 1167338. DOI 10.1017/S0017089500008764.

[Bu2] - Conjugate loci of totally geodesic submanifolds of symmetric spaces, Trans. Amer. Math. Soc. 337 (1993), no. 1, 411-425. MR 1091705. DOI 10.2307/ 2154329.

[Bu3] - Conjugate loci in compact symmetric spaces, Ph.D. dissertation, University of Notre Dame, Notre Dame, Indiana, 1985. MR 2634291.

[C] É. Cartan, Sur certaines formes Riemanniennes remarquables des géométries à groupe fondamental simple, Ann. Sci. Éc. Norm. Supér. (3) 44 (1927), 345-467. MR 1509283.

[Ch1] B.-Y. Chen, A New Approach to Compact Symmetric Spaces and Applications, Katholieke Universiteit Leuven, Louvain, 1987. MR 0952556.

[Ch2] - "Symmetries of compact symmetric spaces" in Geometry and Topology of Submanifolds (Marseille, 1987), World Scientific, Teaneck, N.J., 1989, 38-48. MR 1116130.

[CN1] B.-Y. Chen and T. Nagano, Totally geodesic submanifolds of symmetric spaces, II, Duke Math. J. 45 (1978), 405-425. MR 0487902.

[CN2] - A Riemannian geometric invariant and its applications to a problem of Borel and Serre, Trans. Amer. Math. Soc. 308 (1988), no. 1, 273-297. MR 0946443. DOI $10.2307 / 2000963$.

[EH] J.-H. Eschenburg and E. Heintze, Extrinsic symmetric spaces and orbits of $s$ representations, Manuscripta Math. 88 (1995), 517-524. MR 1362935. DOI 10. $1007 / \mathrm{BF} 02567838$.

[F1] D. Ferus, Immersionen mit paralleler zweiter Fundamentalform: Beispiele und Nicht-Beispiele, Manuscripta Math. 12 (1974), 153-162. MR 0339015.

[F2] - Symmetric submanifolds of Euclidean space, Math. Ann. 247 (1980), 8193. MR 0565140. DOI 10.1007/BF01359868. 
[Ha] A. Hatcher, Algebraic Topology, Cambridge University Press, Cambridge, 2002. MR 1867354.

[He] S. Helgason, Differential Geometry, Lie Groups and Symmetric Spaces, Pure Appl. Math. 80, Academic Press, New York, 1978. MR 0514561.

[HH] W.-T. Hsiang and W.-Y. Hsiang, On the existence of codimension-one minimal spheres in compact symmetric spaces of rank 2, II, J. Differential Geom. 17 (1982), 583-594. MR 0683166.

[HHT] W.-T. Hsiang, W.-Y. Hsiang, and P. Tomter, On the existence of minimal hyperspheres in compact symmetric spaces, Ann. Sci. Éc. Norm. Supér. (4) 21 (1988), 287-305. MR 0956769.

[KN] S. Kobayashi and T. Nagano, On filtered Lie algebras and geometric structures, I, J. Math. Mech. 13 (1964), 875-907. MR 0168704.

[Le] D. S. P. Leung, The reflection principle for minimal submanifolds of Riemannian symmetric spaces, J. Differential Geom. 8 (1973), 153-160. MR 0367872.

[Lo] O. Loos, Symmetric Spaces, II: Compact Spaces and Classification, W. A. Benjamin, New York, 1969. MR 0239006.

[LT] G. Lusztig and J. Tits, The inverse of a Cartan matrix, An. Univ. Timişoara. Ser. Stint. Mat. 30 (1992), 17-23. MR 1329156.

[MQ1] A.-L. Mare and P. Quast, On some spaces of minimal geodesics in Riemannian symmetric spaces, Q. J. Math. 63 (2012), 681-694. MR 2967170. DOI 10.1093/ qmath/har003.

[MQ2] - Bott periodicity for inclusions of symmetric spaces, Doc. Math. 17 (2012), 911-952.

[Ml] J. Milnor, Morse Theory, Ann. of Math. Stud. 51, Princeton University Press, Princeton, 1963. MR 0163331.

[Mi1] S. A. Mitchell, "The Bott filtration of a loop group" in Algebraic Topology (Barcelona, 1986), Lecture Notes in Math. 1298, Springer, Berlin, 1987, 215-226. MR 0928835. DOI 10.1007/BFb0083012.

[Mi2] - Quillen's theorem on buildings and the loops on a symmetric space, Enseign. Math. (2) 34 (1988), 123-166. MR 0960196.

[N1] T. Nagano, The involutions of compact symmetric spaces, Tokyo J. Math. 11 (1988), 57-79. MR 0947946. DOI 10.3836/tjm/1270134261.

[N2] , The involutions of compact symmetric spaces, II, Tokyo J. Math. 15 (1992), 39-82. MR 1164185. DOI 10.3836/tjm/1270130250.

[NS] T. Nagano and M. Sumi, The spheres in symmetric spaces, Hokkaido Math. J. 20 (1991), 331-352. MR 1114410.

[NT1] T. Nagano and M. S. Tanaka, The involutions of compact symmetric spaces, III, Tokyo J. Math. 18 (1995), 193-212. MR 1334718. DOI 10.3836/tjm/1270043621.

[NT2] - The involutions of compact symmetric spaces, V, Tokyo J. Math. 23 (2000), 403-416. MR 1806473. DOI 10.3836/tjm/1255958679.

[Q1] Quast, P., Homotopy of EVII, C. R. Math. Acad. Sci. Paris 350 (2012), 425-426. MR 2922098. DOI 10.1016/j.crma.2012.04.008.

[Q2] , Complex structures and chains of symmetric spaces, Habilitationsschrift, Universität Augsburg, Augsburg, 2010.

[S1] T. Sakai, On the structure of cut loci in compact Riemannian symmetric spaces, Math. Ann. 235 (1978), 129-148. MR 0500710.

[S2] - "Cut loci of compact symmetric spaces" in Minimal Submanifolds and Geodesics (Tokyo, 1977), North-Holland, Amsterdam, 1979, 193-207. MR 0574265 . 
[S3] — Riemannian Geometry, Transl. Math. Monogr. 149, Amer. Math. Soc., Providence, 1996. MR 1390760.

[Se] J.-P. Serre, Complex Semisimple Lie Algebras, Springer, New York, 1987. MR 0914496.

[Tk] M. Takeuchi, On the fundamental group and the group of isometries of a symmetric space, J. Fac. Sci. Univ. Tokyo Sect. I 10 (1964), 88-123. MR 0170983.

[Tn] M. S. Tanaka, Stability of minimal submanifolds in symmetric spaces, Tsukuba J. Math. 19 (1995), 27-56. MR 1346752.

[W] J. A. Wolf, Spaces of Constant Curvature, 5th ed., Publish or Perish, Houston, 1984. MR 0928600.

Institut für Mathematik

Universität Augsburg

Germany

peter .quast@math . uni-augsburg. de 